\title{
Diseño y validación de una escala para medir las actitudes hacia el emprendimiento
}

\section{Design and validation of a scale measuring attitudes toward entrepreneurship}

\author{
Montufar Melo, Antonio Rohman ${ }^{1}$
}

Abstract:

\begin{abstract}
Resumen:
La medición de aquéllos factores vinculados con el emprendimiento (bajo la forma de actitudes) resulta de particular interés para universidades, sociedades de crédito e incubadoras, ya que diversos estudios las ubican como acertados predictores del comportamiento. Así, el objetivo de la presente investigación fue el diseño y validación de una escala para la medición de algunas actitudes relacionadas con el emprendimiento. Se elaboró preliminarmente un cuestionario de 104 reactivos, los cuales se desprendieron de las definiciones teóricas de las variables Autoeficacia percibida, Innovación, Riesgo y Actividades vinculadas al emprendimiento. La muestra estuvo conformada por 189 personas (rango de edad: 17 a 60 años, 98 hombres y 91 mujeres). El análisis factorial determinó la existencia de 10 factores, de los cuales se reportaron 5 por obtener un índice de consistencia interna por encima de .70., cuyas denominaciones finales fueron: Tendencia a las actividades emprendedoras, Percepción de ineficacia, Aversión al riesgo, Propensión al riesgo y Tendencia al cambio. Dichos factores condensaron una muestra de 30 reactivos finales, cuyo alpha de Cronbach alcanzó un valor de .861 .
\end{abstract}

Palabras Clave: Emprendimiento, actitudes hacia el emprendimiento, teoría del comportamiento planificado, diseño y validación de instrumentos, análisis factorial.

${ }^{1}$ Universidad Tecnológica de México - UNITEC México - Campus En Línea

*Correspondencia: armm_15@hotmail.com
The measure of those factors related to entrepreneurship (as attitudes) is interesting for universities, credit companies and business incubators, because there's various investigations that refers them as good behavior predictors. Thus, the aim of this current research was the design and validation of a scale to measure some attitudes related to entrepreneurship. A preliminary version of the questionnaire, with 104 items, was elaborated; those items was built starting from the theoretical definition of the variables Perceived self-efficacy, Innovation, Risk and Activities related to entrepreneurship. 189 persons constituted the sample (age range: 17 to 90 years old, 98 men and 91 women). The factorial analysis decided the presence of 10 factors, of which just 5 was reported because their internal consistency value was above .70 . The final labels that name those 5 factors are Trend to entrepreneurial activities, Perception of inefficiency, Risk aversion, Risk propensity and Tendency to changes. Such factors included a final set of 30 items, which Cronbach alpha reach a value of .861 .

Keywords: Entrepreneurship, attitudes toward entrepreneurship, theory of planned behavior, design and validation of instruments, factorial analysis. 
El emprendimiento, tema de amplio interés en la actualidad dentro de diversos contextos, suele vincularse con la búsqueda de oportunidades de inversión para la creación empresarial en diferentes dimensiones (sea micro, pequeña, o mediana empresa, principalmente). Oviawe (2010) lo define como la "adquisición de habilidades e ideas motivadas por la creación de empleo tanto para uno mismo como para otros. También incluye el desarrollo basado en la creatividad" (p. 114).

La importancia del emprendimiento a nivel social reside en el hecho de que se ha ocupado como un poderoso recurso para apoyar la reducción de la pobreza y así mejorar la economía de ciertas regiones. Los programas sociales de diversas naciones han abierto un espacio para la promoción de las acciones emprendedoras ya que las "empresas de tamaño pequeño y mediano son importantes para la economía" y "los principales agentes del empleo y del crecimiento económico de un país" (Sánchez, 2010, p. 42). En cuanto a la nación mexicana, Durán (2016) refiere que el emprendimiento "surge como una oportunidad para revertir las condiciones de desempleo, y al mismo tiempo, fortalecer el sistema productivo del país" (p. 9).

La medición del emprendimiento atiende a las diversas formas de concebir dicho constructo, ya sea como personalidad emprendedora (Espíritu, Moreno \& Priego, 2012; Muñiz, Suárez-Álvarez, Pedrosa, Fonseca-Pedrero \& García-Cueto, 2014), intenciones emprendedoras (Díaz-García, SáezMartínez \& Jiménez-Moreno, 2015; Espíritu, 2011; Izquierdo \& Buelens, 2008), competencias emprendedoras (Alcaraz \& Villasana, 2015; Correa, Delgado \& Conde, 2011; Espíritu, González \& Alcaraz, 2012; Gómez \& Satizábal, 2011; Sánchez, 2011;) o actitudes hacia el emprendimiento (Espíritu \& Sastre,
2007; Fitzsimmons \& Douglas, 2005; Krauss, 2011; Mora, 2011; Robinson, Stimpson, Huefner \& Hunt, 1991). El análisis de éstas últimas suele trazarse utilizando como base la Teoría de la acción planificada de Ajzen (1991).

De acuerdo a esta teoría, la acción ejercida por un sujeto es precedida por el resultado de una ecuación que integra tres componentes: las actitudes hacia determinado comportamiento, la norma subjetiva, y el control percibido comportamental. El autor añade que estos elementos son "conceptualmente independientes de la intención" (Ajzen, 1991, p. 188), aunque dejando entrever el vínculo entre ellos. Así, la lógica bajo la cual se trazan algunos estudios es que las actitudes preceden a las intenciones, y éstas a la conducta (Elfving, Brännback \& Carsrud, 2009; Fitzsimmons \& Douglas, 2005; Sánchez, 2011), de ahí la importancia de centrarse en dicha secuencia, principalmente en las actitudes, componente que es definido por Ajzen (1991) como "el grado en el que una persona tiene una evaluación, o apreciación, favorable o desfavorable acerca del comportamiento en cuestión" (p. 188). Vinculando este concepto con el emprendimiento, se alude así a una tendencia o inclinación hacia las actividades vinculadas al autoempleo, la cual aumenta la probabilidad de que aquél individuo que la experimente realice la conducta emprendedora, la cual, generalmente, alude al hecho de comenzar un negocio propio.

Las actitudes, como evaluaciones o apreciaciones favorables o desfavorables "que las personas hacen sobre otras personas, ideas o cosas que, técnicamente, reciben la denominación de objetos de actitud" (Morales, Moya, Gaviria \& Cuadrado, 2007, p. 459), suelen concebirse, además, como el agregado de tres tipos de información: 
a) Cognitiva, que involucra pensamientos $\mathrm{y}$ creencias de la persona acerca del objeto actitudinal.

b) Afectiva/Emocional, que refiere sentimientos y emociones asociados al objeto de actitud.

c) Conductual, que recoge intenciones o disposiciones a la acción, además de recuerdos de comportamientos pasados dirigidos hacia el objeto actitudinal (Zanna \& Rempel, 1988).

Teniendo en cuenta todo este conjunto de información acerca de las actitudes, puede comentarse que la importancia de la medición de tales valoraciones relacionadas con el emprendimiento parte de la utilidad que aporta para diversas organizaciones, tales como:

- Incubadoras, para obtener una visión aproximada de aquéllas características depuradas en el sujeto que desee emprender, y aquéllas que merezcan mejorarse.

- Sociedades de crédito, que requieran identificar a quienes posean una mayor capacidad para el emprendimiento.

- Instituciones educativas, de tal forma que se pueda medirse el impacto de ciertos programas para fomentar el emprendimiento, o bien apoyar el diseño de planes de estudio y estrategias didácticas enfocadas a fortalecer dicho constructo. A éste último respecto podría mencionarse que las "escuelas de negocios podrían identificar a sus estudiantes según sus características emprendedoras y aplicar herramientas docentes más efectivas" (Soria-Barreto, ZúñigaJara \& Ruiz, 2016, p. 325) y también que para los centros de educación superior la formación de emprendedores es un motor de desarrollo (Laukkanen, 2000).
- Teniendo en cuenta que el sujeto está en posición de llevar a cabo (o no) la acción emprendedora, dichas organizaciones pueden también considerar la noción de que tales actitudes son capaces de determinar distintos grados de emprendimiento. Valencia, Cadavid, Ríos y Awad (2012) proponen una clasificación flexible y oportuna, que incluye etiquetas tales como las de emprendedor puro, académico-emprendedor, empleado- emprendedor, todero, empleado puro, académico puro y empleado académico, ligadas a otras clasificaciones de mayor jerarquía: emprendedor puro, emprendedor marginal y no emprendedor.

Apuntada esta última cuestión, es posible agregar también la relevancia del concepto de intraemprendimiento, el cual podría definirse como "un tipo de actividad emprendedora llevada a cabo dentro de las organizaciones ya establecidas" (Trujillo \& Guzmán, 2008, p. 38), aludiendo así a una forma de "innovación en empresas existentes" (DíazGarcía et al., 2015, p. 20), también tratada anteriormente por Fitzsimmons y Douglas (2005), y rescatada también más adelante por Romo y Macías (2015). Así, la medición de las actitudes hacia el emprendimiento ya no se limita únicamente a una acción divorciada de las instituciones ya constituidas, sino que también puede concebirse en relación a ellas.

Es necesario acotar que el emprendimiento, siendo un fenómeno social y un constructo de interés para diversas disciplinas, tales como la Administración, la Economía y la Psicología, por nombrar algunas, no es un concepto unidimensional o del cual pueda brindarse un tratamiento aislado. Diversas investigaciones han coincidido en resaltar su 
cualidad multidimensional en cuanto a los diversos factores que, o bien lo constituyen, o se relacionan con él. La Tabla 1, para acompañar este argumento, muestra un conjunto de variables estrechamente vinculadas al emprendimiento.

Como es posible notar, las variables Asunción de riesgo, Autoeficacia, Control percibido, Motivación al logro e Innovación son aquéllas que mayormente suelen ubicarse dentro de las investigaciones acerca del emprendimiento (ver Tabla 1), lo cual refiere su tendencia a fungir como predictores del comportamiento que aquí es concerniente.

Con base en este conjunto de aportaciones es que se desprende el objetivo de la presente investigación, la cual consiste en el diseño y validación de un instrumento para la medición de un conjunto de actitudes relacionadas con el emprendimiento, haciendo uso de la herramienta estadística correspondiente al análisis factorial, por un lado, y el cálculo del coeficiente alpha de Cronbach para cada uno de los factores resultantes.

\section{Tipo de investigación}

La presente investigación es de tipo empírica y de corte transversal dentro del marco cuantitativo, ya que partiendo de la técnica del análisis factorial se busca brindar información acerca de la validez estadística del instrumento. Además de dicho análisis, se procuró la obtención del coeficiente de consistencia interna alpha de Cronbach, buscando así la validación de un instrumento diseñado para la medición de ciertas actitudes hacia el emprendimiento.

\section{Muestra}

La muestra, de tipo no-probabilística, estuvo conformada por 189 personas, residentes de la Ciudad de México y la Zona Metropolita- na, con un rango de edad de 17 a 60 años, 98 del sexo masculino (51.9\% de la muestra) y 91 del femenino (48.1\%). 54 personas eran estudiantes (28.6\%), 118 empleados (62.4\%) y 17 tienen otra ocupación (9\%), como el comercio (empleo informal). En cuanto a estudios alcanzados, 86 habían concluido el nivel superior (45.5\%), 58 el bachillerato $(30.7 \%)$ y 25 contaban con una carrera técnica $(13.2 \%)$. Por último, 84 refirieron haber iniciado alguna vez un negocio propio (44.4\%), mientras que el 105 restante nunca había llevado a cabo alguna acción emprendedora de ese tipo $(55.6 \%)$.

\section{Instrumento}

El instrumento diseñado fue un cuestionario piloto para la medición de ciertas actitudes relacionadas con el emprendimiento, constituido por 104 ítems, cada uno elaborado bajo el formato de escala Likert de cuatro puntos (Muy de acuerdo, De acuerdo, En desacuerdo, Completamente en desacuerdo); se optó por este modelo para evitar las respuestas intermedias ( $\mathrm{Ni}$ de acuerdo ni en desacuerdo) y promover entre los respondientes una postura menos neutra y más tendiente hacia los otros dos polos de respuesta. Tales reactivos se derivaron de una indagatoria previa que contempló los siguientes factores vinculados al emprendimiento, de acuerdo a los estudios revisados: autoeficacia percibida, riesgo e innovación. A este conjunto se le sumó el factor "actividades relacionadas con el emprendimiento", no observado como tal en otros estudios, pero que se consideró relevante para dar cuenta de la tendencia de los individuos por encauzar sus actividades hacia la búsqueda del autoempleo. El criterio bajo el cual se le otorgó tal relevancia al predicho factor se fundamenta en el trabajo de Díaz-García et al. (2015), quienes observaron una mayor incli- 
Tabla 1.

Variables relacionadas con el emprendimiento que mayormente suelen tomarse en cuenta en las investigaciones sobre dicho tema.

Variable Autores

Asunción de riesgo / Aversión al riesgo / Capacidad de asumir riesgos / Propensión al riesgo/ Tolerancia al riesgo / Capacidad para evaluar riesgos

Autoeficacia / Autoeficacia percibida / Viabilidad percibida (autoeficacia)

Autocontrol interno / Control interno / Control percibido interno / Locus de control interno

Motivación al logro / Necesidad de logro

Innovación / Creatividad e innovación / Creatividad y capacidad de innovación / Percepciones de creatividad

Autoestima

Autonomía / Búsqueda de independencia/ Independencia Liderazgo / Capacidad de liderazgo / Autopercepciones de la habilidad para liderar a otros

Personalidad proactiva / Proactividad

Deseo de hacer dinero / Ingreso

Capacidad de esforzarse intensamente / Tolerancia al esfuerzo de trabajo
Cabana-Villca, Cortes-Castillo, Plaza-Pasten, CastilloVergara y Alvarez-Marin (2013); Espíritu (2011); Espíritu y Sastre (2007); Fitzsimmons y Douglas (2005); Mora (2011); Muñiz et al. (2014); Quintero (2007); Rosique, Madrid y García (2012); Saboia y Martín (2006); Sánchez (2010); Santillán, Gaona y Hernández (2015); Segal, Borgia y Schoenfeld (2005); SoriaBarreto et al. (2016); Valencia et al. (2012).

Baum y Locke (2004); Díaz-García et al. (2015); Elfving et al. (2009); Izquierdo y Buelens (2008); Sánchez (2010); Muñiz et al. (2014); Segal et al., (2005); Soria-Barreto et al. (2016); Valencia et al. (2012).

Espíritu (2011); Espíritu y Sastre (2007); Krauss (2011); Lung y Ching (2013); Mora (2011); Muñiz et al. (2014); Robinson et al., (1991); Sánchez (2010); Soria-Barreto et al. (2016).

Athayde (2009); Espíritu (2011); Espíritu y Sastre (2007); Jordán, Serrano, Pacheco y Brito (2016); Krauss (2011); Lung y Ching (2013); Mora (2011); Muñiz et al. (2014); Quintero, (2007); Robinson et al., (1991); Saboia y Martín (2006).

Athayde (2009); Espíritu, Moreno y Priego (2012); Krauss (2011); Lung y Ching (2013); Mora (2011); Muñiz et al. (2014); Robinson et al., (1991); Rosique et al., (2012); Saboia y Martín (2006).

Espíritu et al. (2012); Krauss (2011); Lung y Ching (2013); Mora, (2011); Núñez (2015); Robinson et al. (1991).

Fitzsimmons y Douglas (2005); Muñiz et al. (2014); Saboia y Martín (2006); Santillán et al. (2015).

Athayde (2009); Saboia y Martín (2006); Santillán et al. (2015).

Cabana-Villca et al. (2013); Rosique et al. (2012); Sánchez (2010).

Fitzsimmons y Douglas (2005); Santillán et al. (2015).

Fitzsimmons y Douglas (2005); Santillán et al. (2015)

Fuente: Elaboración propia. 
nación hacia el emprendimiento en aquéllas y aquéllos sujetos que de antemano realizan acciones que implican ingresos por cuenta propia, lo cual habla del "grado en el que el fundador está comprometido con el nuevo negocio en comparación con otras alternativas que pueden ser atractivas para él o ella" ( $p$. 23).

Tomando como base el modelo actitudinal de Zanna y Rempel (1988), el cual propone tres dimensiones que conforman la estructura teórica de las actitudes (cognitiva, afectiva y comportamental) se redactó un banco de 104 ítems que operacionalizaron ciertas situaciones hipotéticas, relativas a cada uno de los factores ya mencionados (autoeficacia percibida, riesgo, innovación y actividades relacionadas con el emprendimiento). Cada uno de dichos reactivos se enfocó en una de las tres dimensiones actitudinales; así, por ejemplo, el ítem "Buena parte de mi tiempo la invierto en algún proyecto que estoy emprendiendo" alude al factor Actividades relacionadas con el emprendimiento, en su dimensión comportamental; el ítem "No me agrada aceptar riesgos" representa al factor Riesgo, en su dimensión Afectiva; el reactivo "Creo que es mejor no estar en posición de tomar decisiones, ya que así uno evita equivocarse" pertenece al factor Autoeficacia percibida, en su dimensión cognitiva; y así por el estilo.

Las definiciones constitutivas de cada uno de los factores, las cuales brindaron el punto de partida para la construcción del banco de ítems, son las siguientes:

Autoeficacia percibida: La autoeficacia se define como "la convicción de tener las habilidades para organizar y producir resultados esperados" (Soria-Barreto et al., 2016, p. 327), y funge como un poderoso conductor del comportamiento orientado a metas (Baum
\& Locke, 2004). Dicha convicción, que la persona posee sobre sus capacidades para la ejecución de determinadas acciones, se vincula con los juicios acerca de qué tan bien puede la persona ejecutar las acciones requeridas de acuerdo a futuras situaciones (Bandura, 1982).

Como puede notarse, no se operacionaliza directamente el constructo de la autoeficacia, sino que se complementa con el adjetivo percibida para hacer énfasis en la noción de juicio o convicción, a modo de creencia, presente en las citas ya comentadas. Esto bajo la influencia y respaldo, tanto de Elfving et al. (2009), que a este respecto comentaron que tal factor "determina lo que la persona cree que puede [cursivas añadidas] hacer" (p. 31), como de Segal et al. (2005), quienes indicaron que para mejorar la actividad emprendedora, es importante fomentar la percepción de autoeficacia antes que otros factores.

Riesgo: Cuando se habla del factor Riesgo en las investigaciones sobre emprendimiento, usualmente se hace alusión a la tendencia de las personas para aceptar ciertos niveles de inseguridad en sus acciones, los cuales facilitarán la consecución de una meta que implica mejores beneficios, en contraste con las posibles consecuencias negativas (Moore \& Gullone, 1996). Espíritu y Sastre (2007), a este respecto, comentan lo siguiente: "una persona que arriesga se puede definir como aquélla que está orientada en aprovechar las oportunidades en contextos inciertos, lo cual está estrechamente relacionado con el concepto de emprendedor" (p. 102).

Innovación: Este factor "permite el desarrollo de nuevos productos, servicios y modelos de negocios en las organizaciones" (Vesga, 2009, p. 1). En la presente investigación, la definición más acorde a su diseño y propósi- 
tos la situó como la actitud que un individuo tiene hacia la búsqueda de nuevas formas de hacer las cosas, lo cual lo mueve, en diversas ocasiones, a no seguir instrucciones; todo esto motiva la evitación de actividades que generan escaso aprendizaje (Mora, 2011).

Actividades relacionadas con el emprendimiento: Una actitud positiva hacia las actividades vinculadas al emprendimiento, de acuerdo a Rosique et al. (2012) puede estar influida por una imagen (también positiva) con respecto a las personas que se consideran emprendedoras. Así, esta variable contempla, de modo más específico, la apreciación que guarda el sujeto acerca de la ejecución de dicho constructo: de llevar a la praxis lo que implica ser un emprendedor. Díaz-García et al. (2015), hablan de un

grado en el que el fundador está comprometido con el nuevo negocio en comparación con otras alternativas que pueden ser atractivas para él o ella, y cuánto está dispuesto/a a sacrificar para conseguir crear su propia empresa, su intención de invertir tiempo y recursos. (p. 23).

\section{Procedimiento}

El cuestionario fue aplicado entre los meses comprendidos desde marzo de 2017 a julio de 2019 en diversas instituciones de la Zona Metropolitana, la cual comprende la Ciudad de México y algunos municipios del Estado de México. Las aplicaciones fueron espaciadas, dependiendo de la posibilidad de realizarlas en cada institución. En marzo de 2017 se realizó el levantamiento de datos, de forma colectiva y guiado por un aplicador, en la sede de InnovaUNAM en la Facultad de Arquitectura (particularmente el centro de Investigaciones en Diseño Industrial), en una de las aulas destinadas a la impartición de los talle- res sobre emprendimiento; la duración de las sesiones fue de treinta minutos, aproximadamente, y éstas se llevaron a cabo alrededor del mediodía. Hacia mayo de ese mismo año fue posible hacerlo en la Torre de Tlatelolco UNAM, en un salón reservado para los talleres, también de forma colectiva y por medio de un aplicador; el tiempo aproximado de aplicación fue de media hora, y alrededor de las catorce y las quince horas. Los meses siguientes y hasta mediados del año posterior (2018), el instrumento fue entregado por un aplicador, para su llenado de forma individual, a empleadas y empleados de las siguientes agencias del Ministerio Público de la Ciudad de México: Gustavo A. Madero 3, Gustavo A. Madero 7, Gustavo A. Madero 7, Iztapalapa 1, Iztapalapa 4, Iztapalapa 9, y también la Fiscalía de delitos sexuales, la Fiscalía de niñas, niños y adolescentes y el Búnker; la duración aproximada para la contestación del instrumento fue de treinta minutos aproximadamente, y se realizó en las oficinas donde laboran las y los empleados, durante su hora reservada para la ingesta de alimentos, que va desde las quince hasta las diecisiete horas. Hacia mediados del año 2018 y hasta julio de 2019 se realizó el levantamiento de datos en la Universidad Tecnológica de México, Campus Atizapán, Estado de México, tanto a estudiantes como a docentes y empleadas y empleados administrativos, en algunos casos de forma colectiva (en las aulas destinadas a clase) y otros de manera individual (en el aula docente), siempre asistidos por un aplicador; el tiempo aproximado para la contestación del instrumento oscilaba alrededor de la media hora, y en un horario ubicado entre las nueve y las trece horas. Durante los mismos meses, y para aumentar el tamaño de la muestra, algunos estudiantes de la predicha institución permitieron establecer el contacto con empre- 
Tabla 3.

Niveles de adquisición de las acciones simbólicas con sus indicadores

Niveles Indicadores

Acciones con objetos sustitutos

Acciones sin apoyo de objetos

Mención de estados y propiedades imaginarias y realización de juego animado

Asignación y representación de roles y habla protagonizada

Nota. Elaboración propia

sas pequeñas, algunas ubicadas en los alrededores del campus y otras en los municipios de Tlalnepantla y Naucalpan de Juárez. El llenado de los cuestionarios, en estos últimos casos, fue de forma individual, guiado por el aplicador; la duración también se mantuvo en un lapso de treinta minutos. En ningún caso se utilizó enmascaramiento.

El análisis de los datos recabados se realizó mediante el software SPSS en su edición vigésima, en el mes de agosto del año 2019, utilizando la técnica del análisis de componentes principales con rotación ortogonal Varimax.

\section{Resultados}

Posterior a la realización del análisis en el software SPSS, se obtuvo que el valor inicial de la medida de adecuación muestral KaiserMayer-Olkin, después de un primer análisis, resultó en .690, el cual sugirió que, por ser mayor a .50, puede utilizarse el análisis factorial con los datos muestrales. La revisión de los valores diagonales de la matriz de correlaciones anti-imagen permitió observar cuáles de los reactivos podían depurarse por hallarse
El niño transfiere el significado de un objeto a otro, utilizándolo de forma representativa simbólica.

En la ausencia del objeto, el niño lo realiza un gesto que representa al objeto en sí mismo o a una acción.

El niño utiliza su lenguaje para la creación de la situación lúdica y para la reproducción de diálogos entre las muñecas y peluches.

El niño distribuye los roles y cuando asume su rol utiliza expresión verbal coherente con el mismo. mayormente alejados de la unidad (se eliminaron aquéllos cuyo valor estaba por debajo de .80; dejando un total de 42 reactivos). Éste último valor (.80) se elige apegándose al criterio expuesto por Frías-Navarro y Soler (2012): "Los valores de la diagonal de di-cha matriz de correlaciones deben tener valores próximos a uno" (p. 55).

Un segundo análisis con los 42 reactivos que permanecieron después del primero, elevó el valor de la medida de adecuación a .861 (prueba de esferidad de Bartlet: 3463.425; $d f=861, \mathrm{p}<.000)$. El método empleado fue el de extracción de componentes principales, más la rotación Varimax. La regla Kaiser-Guttman, de valores propios superiores a 1, permitió la identificación de un total de 10 factores que explicaron el $62.55 \%$ de la varianza acumulada de respuesta al test, de los cuales se reportan sólo los cinco primeros factores (que incluyen un total final de 30 reactivos), dado que el alpha de Cronbach de cada uno de los cinco últimos estuvo por debajo del .70; Campo-Arias y Oviedo (2008), por ejemplo, refieren que la consistencia interna "se considera aceptable cuando se encuentra entre 0.70 y 0.90 " (p. 831). El total de 
la varianza acumulada de esos cinco factores a reportar fue de $48.438 \%$, y el peso factorial de cada uno de los reactivos que los componen estuvo por encima del .40 .

El factor 1 fue denominado Tendencia a las actividades emprendedoras (ver Tabla 2), quedando conformado por 8 ítems, cuyo alpha de Cronbach alcanzó un valor de .840; el factor 2, Percepción de ineficacia (ver Tabla 3), se apegaría a la noción de validez divergente, estipulando que aquéllos sujetos que puntúen bajo en este conjunto de reactivos (6 en total) mostrarán una mejor actitud hacia el emprendimiento (alpha de Cronbach,
.793); el factor 3, rotulado como Aversión al riesgo (ver Tabla 4), establece una clara diferencia con los ítems del cuarto factor, nombrado Propensión al riesgo (ver Tabla 5); el primero de este par quedó conformado por 6 reactivos (alpha de Cronbach, .836), mientras que el segundo por 5, y un valor de consistencia interna de .795; el último factor se denominó Tendencia al cambio (ver Tabla 6), 5 ítems lo conformaron y presentó un alpha de Cronbach de .727. Cada uno de los ya mencionados coeficientes se esquematiza en la Tabla 7 .

Tabla 2

Cargas factoriales de los reactivos correspondientes al primer factor, denominado Tendencia a las actividades emprendedoras

\begin{tabular}{lc}
\hline \multicolumn{1}{c}{ Reactivo } & Carga factorial \\
\hline $\begin{array}{l}\text { Buena parte de mi tiempo la invierto en algún proyecto que estoy empren- } \\
\text { diendo }\end{array}$ & .784 \\
He emprendido proyectos para generar dinero, aunque aquéllos no sean & .702 \\
muy grandes & .685 \\
Puedo emprender un nuevo negocio y salir airoso en tal empresa & .609 \\
Tomo cursos o me capacito para crear mi propia empresa & .538 \\
Me considero capaz de dirigir una empresa pequeña & .531 \\
Soy capaz de tener personal a mi mando & .512 \\
Me agrada descubrir oportunidades de negocio & .507 \\
Me gustaría tener empleados, aun teniendo en cuenta la responsabilidad & \\
\hline
\end{tabular}

Fuente: Elaboración propia.

Tabla 3

Cargas factoriales de los reactivos correspondientes al segundo factor, denominado Percepción de ineficacia.

\begin{tabular}{lc}
\multicolumn{1}{c}{ Reactivo } & Carga factorial \\
\hline Si finalizo algo, es más bien por el temor a las consecuencias de no haberlo & .758 \\
acabado & .733 \\
$\begin{array}{l}\text { A los primeros intentos de completar una tarea, si no lo consigo, comienzo } \\
\text { mejor con otra actividad }\end{array}$ & .720 \\
Creo que es mejor no estar en posición de tomar decisiones, ya que así uno & .528 \\
evita equivocarse & .467 \\
Generalmente los demás toman decisiones por mí & .415 \\
Dependo mucho de otros para hacer las cosas que me he propuesto & \\
Me desagrada todo el conjunto de procesos que implican crear una empresa &
\end{tabular}

Fuente: Elaboración propia. 
Tabla 4

Cargas factoriales de los reactivos correspondientes al tercer factor, denominado Aversión al riesgo.

Reactivo

Carga factorial

Me preocupo con mucha facilidad, por eso prefiero evitar situaciones ries-

.781 gosas

No me agrada aceptar riesgos

Me desanima la probabilidad de poner en riesgo mi capital si emprendiera

algún proyecto

Crear una empresa es un asunto que no va conmigo

A la hora de pensar en nuevas ideas, prefiero que no me tomen en cuenta

Ser una persona que corre riesgos es más una debilidad que una fortaleza

Fuente: Elaboración propia.

Tabla 5

Cargas factoriales de los reactivos correspondientes al cuarto factor, denominado Propensión al riesgo.

\begin{tabular}{lc}
\hline \multicolumn{1}{c}{ Reactivo } & Carga factorial \\
\hline El riesgo que implica hacer algo nuevo me alienta a hacerlo & .758 \\
La verdad es que comúnmente asumo riesgos & .747 \\
Los riesgos que promete el comenzar un nuevo proyecto me motivan a inten- & .591 \\
tarlo & .546 \\
Me gusta aventurarme en nuevos proyectos, muy a pesar del factor fracaso & .435 \\
$\begin{array}{l}\text { Me agrada estar en constante cambio, aunque ello implique algún tipo de } \\
\text { riesgo }\end{array}$ & \\
\hline
\end{tabular}

Fuente: Elaboración propia.

Tabla 6

Cargas factoriales de los reactivos correspondientes al quinto factor, denominado Tendencia al cambio.

Reactivo $\begin{gathered}\text { Carga } \\ \text { factorial }\end{gathered}$

Disfruto haciendo las cosas de diferente modo cada vez que me es posible ha- $\quad .798$ cerlo

Experimento una sensación de logro cuando descubro una nueva forma para resolver algún problema

Me motiva demasiado tener la oportunidad de llevar a cabo las actividades de modo diferente a como usualmente se hace

Me alegra cuando puedo trabajar con personas innovadoras, a las que les atraen los cambios

Creo que emprender un negocio es una buena opción para obtener ingresos

Fuente: Elaboración propia. 
Tabla 7

Coeficientes alpha de Cronbach de cada uno de los factores del instrumento.

\begin{tabular}{ccccc}
\hline $\begin{array}{c}\text { Factor 1 } \\
\begin{array}{c}\text { Tendencia a las } \\
\text { actividades em- } \\
\text { prendedoras }\end{array}\end{array}$ & $\begin{array}{c}\text { Factor 2 } \\
\text { Percepción de } \\
\text { ineficacia }\end{array}$ & $\begin{array}{c}\text { Factor 3 } \\
\text { Aversión al } \\
\text { riesgo }\end{array}$ & $\begin{array}{c}\text { Factor 4 } \\
\text { Propensión al } \\
\text { riesgo }\end{array}$ & $\begin{array}{c}\text { Factor 5 } \\
\text { Tendencia al } \\
\text { cambio }\end{array}$ \\
\hline .840 & .793 & .836 & .795 & .727 \\
\hline
\end{tabular}

Fuente: Elaboración propia.

\section{Discusión}

La congruencia del análisis factorial en cuanto a las dimensiones propuestas inicialmente puede notarse con respecto a aquéllos factores que continuaron hasta el reporte final (aquéllos cuyo coeficiente de consistencia interna estuvo por encima de .70). Así, el factor Tendencia a las actividades emprendedoras se derivó de forma directa de aquél denominado originalmente Actividades relacionadas con el emprendimiento. La relevancia de incluir un factor de este tipo, no contemplado con tanta frecuencia a diferencia de los otros, encuentra un eco en los resultados de algunas investigaciones, preocupadas también por la dinámica de las variables ligadas al emprendedurismo. Pulka, Rikwentishe e Ibrahim (2014), enfatizaron la necesidad de enfocarse en mejorar la percepción que las y los estudiantes tienen con respecto a las actividades emprendedoras (claras representantes del componente comportamental de las actitudes) ya que implican habilidades procedimentales que en muchas ocasiones conllevan a una evaluación negativa por parte del posible emprendedor; si bien los aspectos cognitivo $\mathrm{y}$ afectivo pueden ser valorados positivamente (dado que son supuestos basados más bien en creencias o emociones, y no en hipótesis factuales), al no ser así con las intenciones o anticipaciones de un acto, la o el estudiante puede tener una idea falsa o errónea de lo que implica emprender. Segal et al. (2005), más directos en cuanto al factor que ahora es con- cerniente, comentaron: "Los individuos evalúan si poseen el conocimiento, las destrezas y las habilidades requeridas para realizar las tareas y actividades necesarias para convertirse en un emprendedor" (p. 7). Por último, no está de más destacar lo apuntado por Ajzen y Fishbein (2005), quienes reseñaron la distinción que hay entre las actitudes generales hacia determinado objeto, y las actitudes hacia la ejecución de cierta conducta; dicha distinción se halla en lo siguiente: éstas últimas tienen mayores probabilidades de resultar en acción.

Con respecto al factor Percepción de ineficacia, éste se derivó de los ítems negativos de Autoeficacia percibida (aquéllos que manifestaban la falta de pericia del sujeto para realizar diligencias vinculadas al quehacer emprendedor); a este respecto puede enfatizarse, como ya se comentaba anteriormente, la noción de Validez divergente, dado que se anticipa que aquéllas personas con una buena actitud hacia el emprendimiento, obtendrán puntajes bajos en los reactivos de esta dimensión.

Aversión al riesgo y Propensión al riesgo se derivaron del factor original Riesgo, estando constituido el primero por aquéllos reactivos negativos (rechazo al riesgo), mientras que el segundo compendió algunos cuyas afirmaciones revelaban la inclinación por situaciones riesgosas. Soria-Barreto et al. 
(2016), en relación a este factor, habían hecho el siguiente apunte, el cual bien puede justificar por qué no está de más hacer una división de la dimensión "Riesgo" en cuanto a sus dos grandes facetas, la aversión y la propensión: "la principal característica distintiva de los estudiantes emprendedores es su grado de aversión personal a actuar en oportunidades de negocio en condiciones de incertidumbre, por lo que es sobre esta variable en la que debería centrarse el esfuerzo educativo." ( $p$. $325)$.

Por último, el factor Tendencia al cambio fue el derivado directo de Innovación, tras condensar los ítems que operacionalizaron situaciones referidas a la flexibilidad (en lugar de la rigidez) en la forma de realizar las actividades cotidianas y laborales.

En el presente caso se brinda una aproximación desde el abordaje de las actitudes, ya que, como estipulaban Robinson et al. (1991) desde hace varios años, la perspectiva actitudinal ofrece un modelo más flexible, específico y dinámico para el estudio del emprendimiento, a diferencia, por ejemplo, de la perspectiva propia de la personalidad emprendedora, criticada por considerarse más bien anticuada (Delmar, 2006).

A modo de cierre, no resta sino enfatizar la necesidad de continuar con investigaciones de este tipo pero a mayor escala. Tal como se mencionaba en el apartado correspondiente a las características de la muestra, ésta estuvo conformada por residentes de la Zona metropolitana. No debe descuidarse la importancia de contar con datos de los habitantes de otros estados, para conformar un instrumento más robusto en cuanto a esa característica. Si bien el muestreo fue de tipo no -probabilístico, esta adolescencia se respalda en el hecho de que se trata de un estudio que busca constituir la "materia prima para investigaciones más precisas" (Hernández, Fernández \& Baptista, 2014, p. 176).

Así mismo, queda pendiente el tratamiento y examen de los otros factores que las investigaciones también observan como representantes principales del emprendimiento (control percibido interno, motivación al logro, autoestima, liderazgo, proactividad, por mencionar algunos).

\section{Referencias}

Alcaraz, R., \& Villasana, M. (2015). Construcción y validación de un instrumento para medir competencias emprendedoras. Trabajo presentado en el Decimonoveno Congreso Internacional de Investigación en Ciencias Administrativas, Durango, Dgo. Resumen recuperado de http:// acacia.org.mx/busqueda/pdf/

CONSTRUCCION_Y _VALIDACION_DE_UN_INSTRUMENTO_P ARA_MEDIR_COMPETENCIAS_EMPRENDEDŌRAS.pdf

Athayde, R. (2009). Measuring enterprise potential in young people. Entrepreneurship Theory \& Practice, 33(2), 481-500. doi: 10.1111/j.15406520.2009.00300.x

Baum, J., \& Locke, E. (2004). The relationship of entrepreneurial traits, skill and motivation to subsequent venture growth. Journal of Applied Psychology, 89, 587-598. Recuperado de https:// pdfs.semanticscholar.org/5f59/58ad81958dfe17 e07122de98 adac1521d357.pdf

Cabana-Villca, R., Cortes-Castillo, I., Plaza-Pasten, D., Castillo-Vergara, M., \& Alvarez-Marin, A. (2013). Análisis de las capacidades emprendedoras potenciales y efectivas en alumnos de centros de educación superior, Journal Technology Management and Innovation, 8(1), 65-75. Recuperado de http://www.scielo.cl/pdf/jotmi/v8n1/ art07.pdf

Campo-Arias, A. \& Oviedo, H. (2008). Propiedades psicométricas de una escala: la consistencia interna. Revista de Salud Pública, 10(5), 831839. Recuperado de https://www.redalyc.org/ $\mathrm{pdf} / 422 / 42210515 . \mathrm{pdf}$ 
Correa, Z., Delgado, C., \& Conde, Y. (2011). Formación en emprendimiento en estudiantes de la carrera de administración de empresas en la Universidad Pública de Popayán. Revista Escuela de Administración de Negocios, 71, 4051. Recuperado de http://www.redalyc.org/ pdf/206/20623157004.pdf

Delmar, F. (2006). The psychology of the entrepreneur. En S. Carter \& D. Jones-Evans (Eds.), Enterprise and small business. Principles, practice and policy (pp. 152-175). Essex: Pearson Education Limited.

Díaz-García, C., Sáez-Martínez, F., \& JiménezMoreno, J. (2015). Evaluación del impacto del programa educativo "Emprendedores" en la intención emprendedora de los participantes. RUSC. Universities and Knowledge Society Journal, 12(3), 17-31. doi: 10.7238/ rusc.v12i3.2146

Durán, J. (2016). Emprendimiento juvenil en México. Informe nacional. Recuperado de https:// www.incae.edu/sites/default/files/ reporte_nacional__-_mexico_final_corregido. pdf

Elfving, J., Brännback, M., \& Carsrud, A. (2009). Toward a contextual model of entrepreneurial intentions. En A. Carsrud, \& M. Brännback (Eds.), Understanding the Entrepreneurial Mind (pp. 23-33). doi: 10.1007/978-1-4419-0443-0_2

Espíritu, R., \& Sastre, M. (2007). La actitud emprendedora durante la vida académica de los estudiantes universitarios. Cuadernos de Estudios Empresariales, 17, 95-116. Recuperado de https:// www.researchgate.net/ publication/27594217_La_actitud_ emprendedora_durante_la_vida_academica_de_los_estud iantes_universitarios

Espíritu, R. (2011). Análisis de la intención emprendedora en estudiantes universitarios a través de los rasgos de personalidad. Multiciencias, 1, 65-75. Recuperado de https://www.redalyc.org/ pdf/904/90418851009.pdf

Espíritu, R., González, R., \& Alcaraz, E. (2012). Desarrollo de competencias emprendedoras: Un análisis explicativo con estudiantes universitarios. Cuadernos de Estudios Empresariales, 22, 2953. doi: http://dx.doi.org/10.5209/rev CESE.2012.v22.44644

Espíritu, R., Moreno, H., \& Priego, H. (2012). Rasgos de personalidad innovación y autoestima en la intención emprendedora de estudiantes universitarios. Multiciencias, 12, 52-58. Recuperado de https://www.redalyc.org/ pdf/904/90431109008.pdf
Fitzsimmons, J., \& Douglas, E. (2005). Entrepreneurial attitudes and entrepreneurial intentions: a cross-cultural study of potential entrepreneurs in India, China, Thailand and Australia. Trabajo presentado en la conferencia de BabsonKauffman Entrepreneurial Research, Wellesley, MA.

Frías-Navarro, D. \& Soler, M. (2012). Prácticas del análisis factorial exploratorio (AFE) en la investigación sobre conducta del consumidor y marketing. Suma Psicológica, 19(1), 47-58. Recuperado de https://www.uv.es/ friasnav/ FriasNavarroMarcopsSoler.pdf

Gómez, M., \& Satizábal, K. (2011). Educación en emprendimiento: fortalecimiento de competencias emprendedoras en la Pontificia Universidad Javeriana Cali. Economía, gestión y desarrollo, 11, 121-151. Recuperado de http:// revistaeconomia.puj.edu.co/html/articulos/ Numero_11/SATIZABAL.pdf

Hernández, R., Fernández, C., \& Baptista, P. (2014). Metodología de la investigación. México: McGraw Hill.

Izquierdo, E., \& Buelens, M. (2008). Competing models of entrepreneurial intentions: the influence of entrepreneurial self-efficacy and attitudes. Trabajo presentado en la Internationalizing Entrepreneurship Education and Training, IntEnt2008 Conference, Oxford, Ohio, USA.

Jordán, J., Serrano, B., Pacheco, A., \& Brito, L. (2016). Capacidad emprendedora desde la perspectiva de género. Opción, 32(81), 238-261. Recuperado de http://www.redalyc.org/ articulo.oa? id $=31048807012$

Krauss, C. (2011). Actitudes emprendedoras de los estudiantes universitarios: El caso de la Universidad Católica del Uruguay. Dimensión Empresarial, 9(1), 28-40. Recuperado de https:// dialnet.unirioja.es/descarga/articulo

Laukkanen, M. (2000). Exploring alternative approaches in high-level entrepreneurship education: creating micromechanisms for endogenous regional growth. Entrepreneurship and Regional Development, 12, 25-47. Recuperado de https:// www.researchgate.net/

publica-

tion/235936851_Exploring_Alternative_Approa ches_in_High-

Lev-

el_Entrepreneurship_Education_Creating_Micr o-Mechanisms__ for ${ }_{-}$Endogenous_Regional_Growth

Lung, K., \& Ching, S. (2013). An exploratory study on the relationship between entrepreneurial attitude 
and firm performance. Human Resource Management Research, 3(1), 34-38. doi: 10.5923/ j.hrmr.20130301.07

Moore, S., \& Gullone, E. (1996). Predicting adolescent risk behavior using a personalized cost-benefit analysis. Journal of Youth and Adolescence, 25 (3), 343-358. doi: 10.1007/BF01537389

Mora, R. (2011). Estudio de actitudes emprendedoras con profesionales que crearon empresa. Revista Escuela de Administración de Negocios, 71, 7083. Recuperado de https://www.redalyc.org/ pdf/206/20623157006.pdf

Morales, J., Moya, M., Gaviria, E., \& Cuadrado, I. (2007). Psicología social. Madrid: McGraw Hill.

Muñiz, J., Suárez-Álvarez, J., Pedrosa, I., FonsecaPedrero, E., \& García-Cueto, E. (2014). Enterprising personality profile in youth: Components and assessment. Psicothema, 4, 545-553. doi: $10.7334 /$ psicothema2014.182

Núñez, M. (2015). Autoestima y habilidades emprendedoras en estudiantes de nivel medio superior. Revista Electrónica de Psicología Iztacala, 18 (1), 358-379. Recuperado de http:// www.revistas.unam.mx/index.php/repi/article/ view/48206/43336

Oviawe, J. I. (2010). Repositioning nigerian youths for economic empowerment through entrepreneurship education. European Journal of Educational Studies, 2(2), 113-118. Recuperado de https://www.academia.edu/2071962/

Repositioning_Nigerian_youths_ for_economic_empowerment_through_entrepre neurship_education

Pulka, M., Rikwentishe, R., \& Ibrahim, B. (2014). An evaluation of students' attitude towards entrepreneurship education in some selected universities in North East Nigeria. Global Journal of Management and Business Research, 14(8), 19.

Quintero, C. (2007). Generación de competencias en jóvenes emprendedores. Recuperado de: http:// www.usmp.edu.pe/facarrhh/ primer_congreso_ippeu_boletin/ppts/Carlos Quinteros.pdf

Robinson, P., Stimpson, D., Huefner, J., \& Hunt, H. (1991). An attitude approach to the prediction of entrepreneurship. Entrepreneurship Theory and Practice, 15(4), 13-31. Recuperado de https://www.researchgate.net/

publication/270820261_An

Atti-

tude_Approach_to_the_Prediction_of_Entrepre neurship
Romo, L., \& Macías, G. (2015). La cultura emprendedora con equidad de género a través del lenguaje de los docentes en la enseñanza de cursos de emprendedurismo casos. En F. Ojeda \& I. Carrillo (Eds.), La subversión narrativa y mediaciones para la construcción de un contradiscurso femenino (pp. 107-139). Recuperado de https://www.researchgate.net/profile/

Jose_Felipe_Hidalgo/

publica-

tion/293654371_La_subversion_narrativa_y_m ediaciones_para_la_construccion_de_un_contra discurso_femenino/

links/56ba688f08ae6a0040adfdfb/La-

subversion-narrativa-y-mediaciones-para-laconstruccion-de-un-contradiscursofemenino.pdf?origin=publication_detail

Rosique, M., Madrid, A., \& García, D. (2012). Entrepreneurial attitudes: An empirical analysis in secondary education students. Uma Ética para Quantos, 33(2), 81-87. Recuperado de https:// pdfs.semanticscholar.org/ c3e8/082c0355a348d9bcb390674e03 897946e034.pdf

Saboia, F., \& Martín, N. (2006). Los rasgos psicológicos del emprendedor y la continuidad del proyecto empresarial: un estudio empírico de los emprendedores brasileños. REAd, Revista Eletrônica de Administração, 12(2). Recuperado de http://www.redalyc.org/ pdf/4011/401137451002.pdf

Sánchez, J. (2010). Evaluación de la personalidad emprendedora: validez factorial del cuestionario de orientación emprendedora (COE). Revista Latinoamericana de Psicología, 1, 41-52. Recuperado de https://www.redalyc.org/pdf/805/ 80515880004.pdf

Sánchez, J. C. (2011). University training for entrepreneurial competencies: Its impact on intention of venture creation. International Entrepreneur Management Journal, 7, 239-254. doi: 10.1007/ s11365-010-0156-x

Santillán, R., Gaona, E., \& Hernández, N. (2015). El perfil del emprendedor que apoyan los fondos de capital privado/capital emprendedor en México. Contaduría y Administración, 60(1). Recuperado de http://www.cya.unam.mx/ index.php/cya /article/view/818/867

Segal, G., Borgia, D., \& Schoenfeld, J. (2005). The motivation to become an entrepreneur. International Journal of Entrepreneurial Behaviour \& Research, 11(1), 42-57. Recuperado de https://www.researchgate.net/ 
publication/228314700_The__ Motivation to Become an Entrepreneur

Soria-Barreto, K., Zúñiga-Jara, S., \& Ruiz, S. (mayo, 2016). Determinantes de la intención emprendedora: Nueva evidencia. Interciencia, 41(5), 325329. Recuperado de http://www.redalyc.org/ articulo.oa? id $=33945552006$

Trujillo, M., \& Guzmán, A. (2008). Intraemprendimiento: una revisión al constructo teórico, sus implicaciones y agenda de investigación futura. Cuadernos de Administración, 21(35), 37-63. Recuperado de http://www.scielo.org.co/pdf/ $\mathrm{cadm} / \mathrm{v} 21 \mathrm{n} 35 / \mathrm{v} 21 \mathrm{n} 35 \mathrm{a} 03 . \mathrm{pdf}$

Valencia, A., Cadavid, L., Ríos, D., \& Awad, G. (2012). Factores que inciden en las intenciones emprendedoras de los estudiantes. Revista Venezolana de Gerencia, 17(57), 132-148. Recuperado de http://www.redalyc.org/ articulo.oa? id $=29021992008$

Vesga, R. (2009). Emprendimiento e innovación en Colombia: ¿Qué nos está haciendo falta? Recuperado de: http://cec.uniandes.edu.co/pdf/ rav.pdf

Zanna, M., \& Rempel, J. (1988). Attitudes: A new look at an old concept. En D. Bar-Tal \& A. W. Kruglanski (Eds.), The social psychology of knowledge (pp. 315-334). Cambridge: Cambridge University Press. 